\title{
Modelling Parametrical Perturbation of Fluctuations of the Power Unit on the Suspender
}

\author{
Igor V. Alekseev ${ }^{1}$, Sergey N. Bogdanov ${ }^{2}$, Pavel V. Safronov ${ }^{3}$, Andrey L. Yakovenko ${ }^{4}$ \\ ${ }^{1}$ Professor, Moscow Automobile and Road Construction State Technical University (MADI), Moscow, Russia, \\ dvs@madi.ru \\ ${ }^{2}$ Professor, Moscow Automobile and Road Construction State Technical University (MADI), Moscow, Russia, \\ dvs@madi.ru \\ ${ }^{3}$ Associate professor, Moscow Automobile and Road Construction State Technical University (MADI), Moscow, \\ Russia,pavel_safronov@mail.ru \\ ${ }^{4}$ Associate professor, Moscow Automobile and Road Construction State Technical University (MADI), Moscow, \\ Russia, iakovenko_home@mail.ru (corresponding author)
}

\begin{abstract}
The technique and results of support taking into account variability of its inertial characteristics of the parts caused by movement inside the body is given in article. The technique is based on use of the equations of Lagrange of the second sort. Methods for calculating the kinetic energy of a piston, connecting rod, crankshaft, and engine block are presented. The values included in the formula are explained. Expressions for calculating linear velocity components for each engine element are shown. The order of receiving system of the equations of Lagrange of the second sort for four the cylinder inline engine is given as an example. Mass and inertial characteristics of the main moving elements were determined by analytical methods based on the working drawings of these elements. The amplitudes of the oscillations of the attachment points of the housing to the elastic element were calculated for a sequence of fixed values of the angular velocity of rotation of the crankshaft in the range $200 \ldots 1000$ $\mathrm{rpm}$. The analysis of the results shows a difference between the calculated characteristics obtained taking into account the mobility of the elements inside the case and in the absence thereof. The differences between the results of the two compared methods are quite fundamental and are not only quantitative, but also qualitative.
\end{abstract}

Key words: amplitude-frequency characteristic, moment of inertia, engine mount, Lagrange's equations.

\section{INTRODUCTION}

The kinematic variability of the crank mechanism, which forms the basis of the engine design, entails the variability of the inertial characteristics of the power unit as a dynamic system. Approximate methods for calculating engine vibrations often ignore this fact. In such cases, it is generally believed that some inertia forces act on an absolutely rigid engine body. The values of this forces are associated with the masses and accelerations of the moving links. The inconsistency of this approach is that the inertia forces are applied not to the moving bodies inside the engine body, but to the center of mass of the engine body itself [4], [5]. In this case, the inertial characteristics of the housing are assumed unchanged. The following describes the calculation of the oscillations of the engine, taking into account the variability of its inertial characteristics.

\section{MATERIALS AND METHODS}

\subsection{The choice of design scheme and calculation method}

As a calculation model, we used a three-dimensional scheme of a four-cylinder in-line engine with four elastic mounting arranged arbitrarily. The block crankcase, the elements of the crank mechanism and pistons were presented in the form of absolutely solid bodies that constitute a cinematically variable structure [7], [8]. The elastic supports were represented as linear stiffeners with parallel connected linear dampers. The geometric parameters that determine the relative position of the centers of mass of parts and the attachment points of elastic supports are shown in Figure 1 and Figure 2.

The designations in the figures have the following meanings: $C p, M p, J p$ - are the center of mass, mass and moment of inertia of the piston, $C s$ - is the center of mass, mass and moment of inertia of the connecting rod, $C v$ - is the center of mass, mass and moment of inertia of the crankshaft, $C k$ - is the center of mass, mass and moment of inertia of the crankcase assembly with the cylinder head.

Depicted in Figure 1 and Figure 2, the design has seven degrees of freedom - its position at an arbitrary moment in time can be uniquely determined by the following independent kinematic parameters:

$q_{1}, q_{2}$ and $q_{3}$ - horizontal (along the $\mathrm{X}$-axis), vertical (along the $\mathrm{Y}$-axis) and longitudinal (along the $\mathrm{Z}$-axis) displacements of the center of inertia of the aggregate; 
Igor V. Alekseev et al., International Journal of Emerging Trends in Engineering Research, 8(6), June 2020, 2719 - 2723

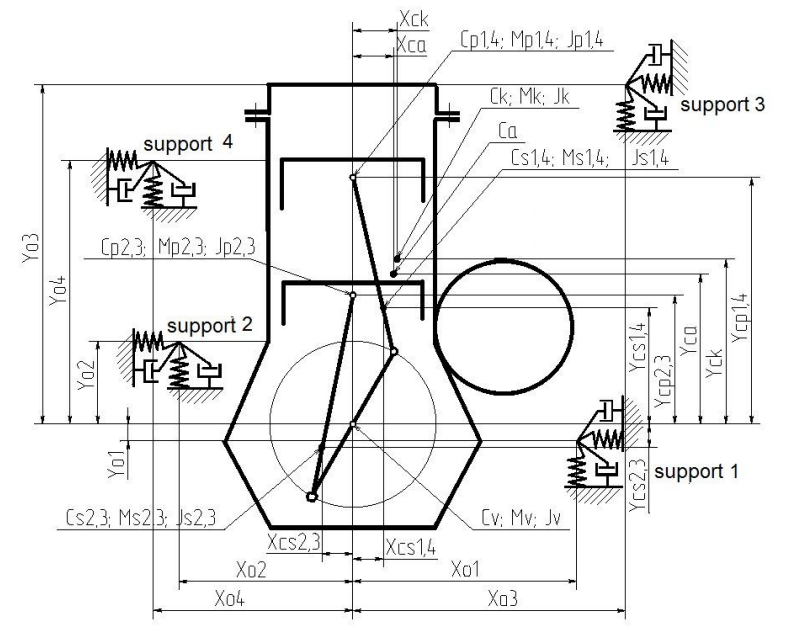

Figure 1: The design scheme of a four-cylinder engine on the suspension - front view

$q_{4}$ - is the angle of deviation of the unit in the XoY plane; $q_{5}$ - is the angle of deviation of the unit in the XoZ plane; $q_{6}$ - is the angle of deviation of the unit in the YoZ plane; $q_{7}$ - is the angle of rotation of the crankshaft of the engine relative to its longitudinal axis.

Assuming that the crankshaft rotates about its axis according to a predetermined law, it is possible to exclude the angle of rotation of the crankshaft from among the free kinematic parameters of the system [1]. Adopting a certain law of change in the angle of crankshaft rotation (CR), excluding this kinematic parameter from the number of freely specified ones and reducing the number of degrees of freedom of the analyzed structure to six, we actually proceed to the analysis of kinematic loading or, in other words, loading by maintaining the given law of change in the angle of the CR. At the same time, those external forces that support the given law of changing the angle of the CR will not explicitly participate in the equations of dynamics obtained subsequently.

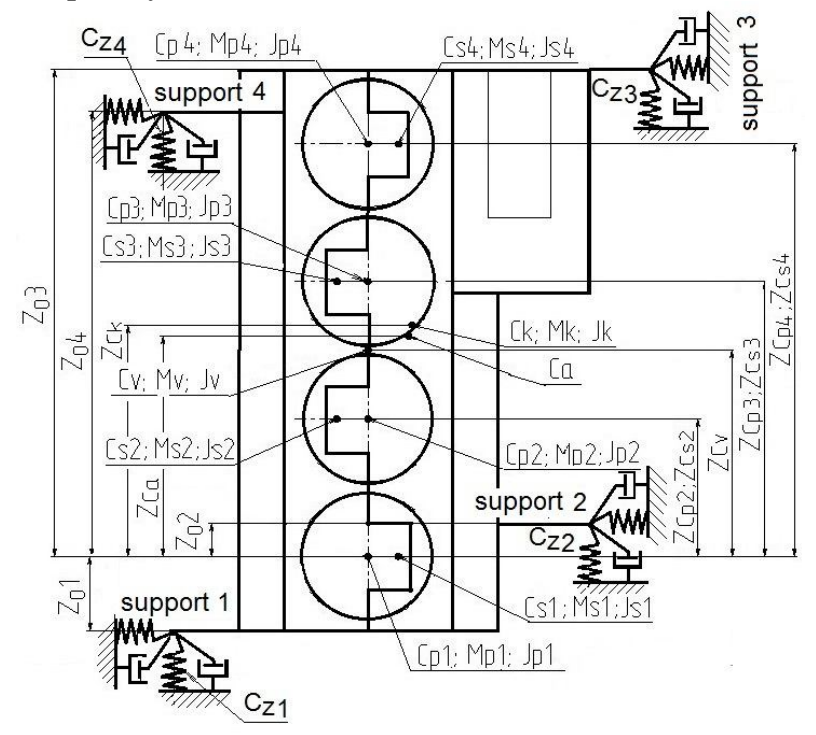

Figure 2: The design scheme of the four-cylinder engine on the suspension - top view
2.2 The derivation of the basic equations of the dynamics of the power unit taking into account the variability of the mass-inertial characteristics of the engine

Depicted in Figure 1 and Figure 2, the design is non-conservative due to the presence of damping elements in it. In order to facilitate the application of the second order Lagrange equations to this construction [2], we assume that there are no damping elements, but instead some forces are applied to the corresponding support points of the crank-case that enter the Lagrange equations as external forces. At the last stage, when the Lagrange equations are obtained, these forces will be given expressions in the form of a product of linear damping coefficients and the elongation of the support (elementary expressed through the basic kinematic parameters and the necessary geometric dimensions) with a minus sign. Thus, the influence of damping in the supports will be fully taken into account.

Lagrange equations of the second kind have the form:

$$
\frac{\partial L}{\partial q_{i}}-\frac{d}{d t}\left(\frac{\partial L}{\partial \dot{q}_{i}}\right)=Q_{i},
$$

where $q_{i}$ - is the generalized displacement (in this case, $q_{1}, q_{2}$ and $q_{3}$ will be the horizontal, vertical and longitudinal displacements of the body center of mass, $q_{4}$ - is the angle of the deflection of the body in the XoY plane, $q_{5}$ - is the angle of deflection of the body in XoZ plane, $q_{6}$ - is the angle of deflection of the body in the YoZ plane).

Lagrange L-function, which is expressed as follows:

$L=T-U$,

where $T$ and $U$ are the potential and kinetic energies of the system, respectively. $Q_{i}$ - is the generalized force corresponding to the $\mathrm{i}$-th generalized displacement (in this case, as indicated above, $Q_{i}=0$ ).

The potential energy of the studied structure is accumulated only in the elastic sup-ports as a result of their deformation. A detailed derivation of the equations for potential energy is presented in [1] and [6].

The kinetic energy of a system is the sum of the kinetic energies of its individual elements:

$T_{a}=T_{K}+T_{V}+\sum_{i=1}^{4} T_{S i}+\sum_{i=1}^{4} T_{P i}$,

where $T_{K}, T_{V}, T_{S i}, T_{P i}$ - are the kinetic energies of the crankcase, crankshaft, connecting rods and pistons, respectively. 
Igor V. Alekseev et al., International Journal of Emerging Trends in Engineering Research, 8(6), June 2020, 2719 - 2723

\section{Kinetic energy of the body}

$T_{K}=\frac{1}{2} \cdot M_{K} \cdot\left(V_{C K x}^{2}+V_{C K y}^{2}+V_{C K z}^{2}\right)+$

$+\frac{1}{2} \cdot\left(J_{K z} \cdot \dot{q}_{4}^{2}+J_{K y} \cdot \dot{q}_{5}^{2}+J_{K x} \cdot \dot{q}_{6}^{2}\right)$,

where $V_{C K x}, V_{C K y}$, and $V_{C K z}$ - are the linear components of the velocity of the center of mass of the housing, which are expressed as follows in terms of the generalized velocities of the system:

$$
\begin{aligned}
& V_{C K x}=\dot{q}_{1}-\left(Y_{C K}-Y_{C A}\right) \cdot \dot{q}_{4}+\left(Z_{C K}-Z_{C A}\right) \cdot \dot{q}_{5} ; \\
& V_{C K y}=\dot{q}_{2}+\left(X_{C K}-X_{C A}\right) \cdot \dot{q}_{4}+\left(Z_{C K}-Z_{C A}\right) \cdot \dot{q}_{6} ; \\
& V_{C K z}=\dot{q}_{3}+\left(X_{C K}-X_{C A}\right) \cdot \dot{q}_{5}+\left(Y_{C K}-Y_{C A}\right) \cdot \dot{q}_{6}
\end{aligned}
$$

\section{Kinetic energy of the crankshaft}

$$
\begin{aligned}
& T_{V}=\frac{1}{2} \cdot M_{V} \cdot\left(V_{C V x}^{2}+V_{C V y}^{2}+V_{C V z}^{2}\right)+ \\
& +\frac{1}{2} \cdot\left(J_{V z} \cdot \omega_{V z}^{2}+J_{V y} \cdot \dot{q}_{5}^{2}+J_{V x} \cdot \dot{q}_{6}^{2}\right),
\end{aligned}
$$

where $V_{C V x}, V_{C V y}$, and $V_{C V z}$ - are the linear components of the velocity of the center of mass of the crankshaft, is the angular velocity of the crankshaft. These quantities are ex-pressed as follows through the generalized speeds of the system:

$$
\begin{aligned}
& V_{C V x}=\dot{q}_{1}+Y_{C A} \cdot \dot{q}_{4}-\left(Z_{C A}-Z_{C V}\right) \cdot \dot{q}_{5} ; \\
& V_{C V z}=\dot{q}_{3}-X_{C A} \cdot \dot{q}_{5}-\left(Y_{C A}-Y_{C V}\right) \cdot \dot{q}_{6} ; \\
& V_{C V y}=\dot{q}_{2}+X_{C A} \cdot \dot{q}_{4}-\left(Z_{C A}-Z_{C V}\right) \cdot \dot{q}_{6} ; \\
& \omega_{V z}=\omega+\dot{q}_{4}
\end{aligned}
$$

\section{Piston kinetic energy}

Assuming the inertia of rotation of the piston relative to the axis of the piston pin to be negligible, the kinetic energy for the piston of the 1st cylinder may be written in the following form

$T_{P 1}=\frac{1}{2} \cdot M_{P 1} \cdot\left(V_{C P 1 x}^{2}+V_{C P 1 y}^{2}+V_{C P 1 z}^{2}\right)+$

$+\frac{1}{2} \cdot\left(J_{P 1 z} \cdot \dot{q}_{4}^{2}+J_{P 1 y} \cdot \dot{q}_{5}^{2}+J_{P 1 x} \cdot \dot{q}_{6}^{2}\right)$,

where $V_{P 1 x}, V_{P 1 y}$ and $V_{P 1 z}$ - are the linear components of the piston speed.

These components are further presented as follows:

$$
\begin{aligned}
& V_{P 1 x}=\dot{q}_{1}-\left(f_{P 1}(\varphi)-Y_{C A}\right) \cdot \dot{q}_{4}-Z_{C A} \cdot \dot{q}_{5} ; \\
& V_{P 1 y}=\dot{q}_{2}+\frac{d}{d t}\left(f_{P 1}(\varphi)\right)-X_{C A} \cdot \dot{q}_{4}-Z_{C A} \cdot \dot{q}_{6} ; \\
& V_{P 1 z}=\dot{q}_{3}-X_{C A} \cdot \dot{q}_{5}+\left(f_{P 1}(\varphi)-Y_{C A}\right) \cdot \dot{q}_{6},
\end{aligned}
$$

where $\mathrm{fP} 1(\varphi)$ - is the current value of the distance of the center of mass of the 1st piston from the axis of the crankshaft
(Figure 3). The last value for the i-th piston is obtained as the sum of the lengths of the projections of the crank and connecting rod on the vertical

$$
f_{P i}(\varphi)=r \cdot \sin \left(\varphi_{i}\right)+l \cdot \cos \left(\beta_{i}\right) \text {, }
$$

where $\beta \mathrm{i}$ - is the angle of deviation of the $\mathrm{i}$-th connecting rod from the vertical

$$
\sin \left(\beta_{i}\right)=\frac{r}{l} \cdot \cos \left(\varphi_{i}\right) ; \varphi_{1,4}=\varphi ; \varphi_{2,3}=\varphi+\pi .
$$

Similarly, taking into account the kinematics of the crank mechanism, it is possible to represent the kinetic energy of the remaining pistons through generalized coordinates.

\section{The kinetic energy of the connecting rod}

When expressing the kinetic energy of the connecting rod, it is necessary to take into account the energy of both translational motion and rotation around the axis of the piston pin. Thus, the kinetic energy for each of the four connecting rods can be expressed as:

$$
\begin{aligned}
& T_{S i}=\frac{1}{2} \cdot M_{S i} \cdot\left(V_{S i x}^{2}+V_{S i y}^{2}+V_{S i z}^{2}\right)+ \\
& +\frac{1}{2} \cdot\left(J_{S i z} \cdot \omega_{S i z}^{2}+J_{S i y} \cdot \dot{q}_{5}^{2}+J_{S i x} \cdot \dot{q}_{6}^{2}\right),
\end{aligned}
$$

where $V_{S i x}, V_{S i y}$ and $V_{S i z}$ - are the linear components of the velocity of the center of mass of the i-th connecting rod; $\omega_{\mathrm{Siz}}$ - is its angular velocity. These values (for the connecting rod of the 1-st cylinder) are expressed as follows:

$$
\begin{aligned}
& V_{S 1 x}=\dot{q}_{1}+\frac{d}{d t}\left[f_{C S 1}(\varphi)_{x}\right]+ \\
& +\dot{q}_{4} \cdot\left[Y_{C A}-f_{C S 1}(\varphi)_{y}\right]-Z_{C A} \cdot \dot{q}_{5} ; \\
& V_{S 1 y}=\dot{q}_{2}+\frac{d}{d t}\left[f_{C S 1}(\varphi)_{y}\right]- \\
& -\dot{q}_{4} \cdot\left[X_{C A}-f_{C S 1}(\varphi)_{x}\right]-Z_{C A} \cdot \dot{q}_{6} ; \\
& V_{S 1 z}=\dot{q}_{3}-\dot{q}_{5} \cdot\left[X_{C A}-f_{C S 1}(\varphi)_{x}\right]- \\
& -\dot{q}_{6} \cdot\left[Y_{C A}-f_{C S 1}(\varphi)_{y}\right] \\
& \omega_{S 1 z}=\dot{q}_{4}+\frac{d}{d t}\left[\beta_{1}(\varphi)\right]=\dot{q}_{4}+\frac{d \beta_{1}}{d \varphi} \cdot \omega
\end{aligned}
$$

where $f_{C S 1}(\varphi)_{x}$ and $f_{C S 1}(\varphi)_{y}$ - are the current values of the horizontal and vertical distances, respectively, from the center of mass of the first connecting rod to the axis of the crankshaft (Figure 3). 
Igor V. Alekseev et al., International Journal of Emerging Trends in Engineering Research, 8(6), June 2020, 2719 - 2723

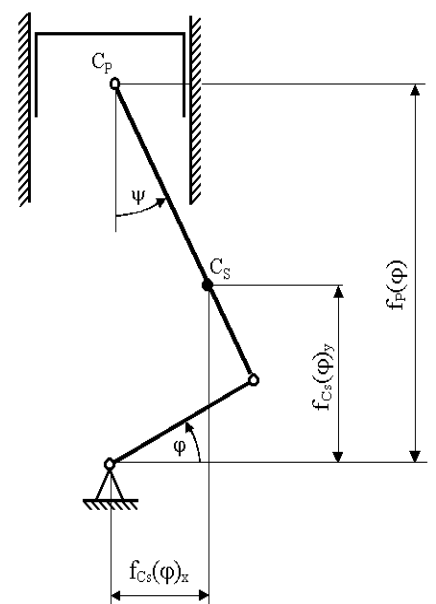

Figure 3: The design scheme of crank mechanism

Similarly, taking into account the kinematics of the crank mechanism, it is possible to represent the kinetic energy of the other connecting rods through generalized coordinates.

The main analytical difficulties of the further solution are associated with cumbersome trans-formations in the expressions of potential and kinetic energies and with their subsequent differentiation by generalized coordinates and time.

These difficulties were overcome through the use of the "EULER" software package, designed to calculate multicomponent mechanical systems, as well as their kinematic and dynamic analysis. The main advantage of working with this software package is a fairly simple method for creating a calculation model that is as close as possible to traditional design, while accurate equations of motion are automatically generated within the framework of classical mechanics. The formed equations of motion are solved by numerical methods [3].

\section{Numerical implementation of powertrain motion analysis}

The described form of the equations made it possible to use the numerical Runge-Kutta method to solve them. The advantage of this method is high accuracy and sufficient computational stability for rounding errors. The error of the method is proportional to the magnitude of the step to the fifth degree [3], which allows changing the magnitude of the step to obtain the necessary accuracy of the solution.

\section{RESULTS}

The effect of mass movement inside engine housing and the method of applying inertial forces on the calculated amplitude-frequency characteristics of the oscillations of the power unit on an elastic suspension was studied. In this case, the law of change of the angle of rotation of the crankshaft corresponded $\omega=$ const. The amplitudes of the oscillations of the attachment points of the housing to the elastic element were calculated for a sequence of fixed values of the angular velocity of rotation of the crankshaft in the range 200...1000 $\mathrm{rpm}$. For each value of the angular velocity of rotation of the crankshaft, the amplitudes of the steady-state oscillations were determined.

Over the entire set of calculations, the dependence of the amplitudes of the forced oscillations on the angular velocity was built. As a prototype, when determining the values of the parameters of the calculation model, the VAZ-2106 engine was chosen. Mass and inertial characteristics of the main moving elements were determined by analytical methods based on the working drawings of these elements.

The stiffness characteristics of the elastic elements of the suspension were determined experimentally. The damping in the suspension elements was set in such a way as to provide an approximate correspondence of the amplitudes of the forced vibrations in the resonance region by the calculated and experimental methods [4]. The admissibility of this approach is determined by the well-known fact of a significant influence of the damping value on the amplitude of the forced oscillations in the resonance region and the practical absence of such an effect in the resonance region.

Figure 4 shows the calculated values of the oscillation amplitudes (A) of the front left support in the vertical direction (along the $\mathrm{Y}$-axis) of the engine speed (n) at idle. The calculations were carried out for the following models with varying degrees of accounting for the variability of inertial characteristics. Model 1 fully takes into account the movement of all parts of the crank mechanism and the effect of this movement on the change in the inertial characteristics of the unit. Model 2 partially takes into account these movements. Often, to simplify the calculations, the rotational movement of the connecting rod is not taken into ac-count. A real connecting rod with distributed mass is replaced by a certain conditional circuit [7], [8], consisting of two concentrated masses connected by a weightless rod. In this case, the dynamic effects caused by the swing of the connecting rod around the piston pin are ignored. Model 3 completely excludes from consideration the changes in the inertial characteristics of the unit. The perturbing force corresponding to the total inertia forces of the reciprocating moving masses is applied to the center of inertia of the power unit. Such schemes are used in the analysis of engine vibrations on a suspension using the frequency method [5].

The analysis of the frequency response shown in Figure 4 shows a significant difference between the calculated characteristics obtained taking into account the mobility of the elements inside the case and in the absence thereof. The differences between the results of the two compared methods are quite fundamental and are not only quantitative, but also 
Igor V. Alekseev et al., International Journal of Emerging Trends in Engineering Research, 8(6), June 2020, 2719 - 2723

qualitative. Despite the identical geometric and mass-inertial characteristics of models 1 and 3 , an increase in the amplitudes of oscillations in the resonance zone is observed.

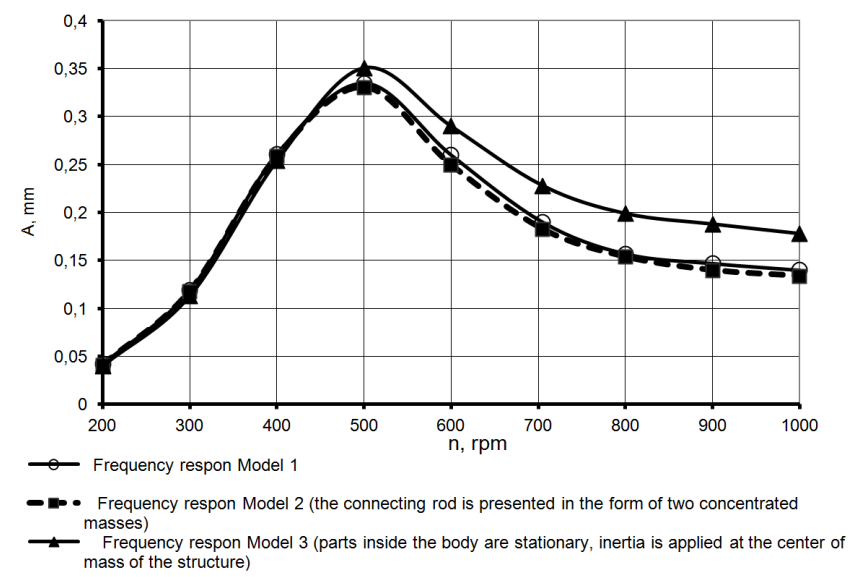

Figure 4: The estimated amplitudes of the vertical movements of the left front support

The shift of the center of mass and the change in the moment of inertia of the power unit leads to an increase in the amplitudes of vertical oscillations in the resonance zone, which is explained by an increase in the coupling of oscillations in all generalized coordinates.

The frequency response of models 1 and 2 is almost the same, which indicates the admissibility of using the equivalent connecting rod circuit when calculating the dynamic behavior of the engine on the suspension at idle.

\section{CONCLUSION}

1. Using the considered calculation method allows us to take into account the influence of the variability of the mass-inertial characteristics of the power unit, due to the kinematic variability of the crank mechanism, on the parameters of power unit oscillations.

2. Comparison of the frequency response of models 1 and 3 allows us to conclude that there is a significant error in the results obtained by applying the resulting inertia to the center of inertia of the power unit.

3. The proposed methodology makes it possible to evaluate the effect of a change in the position of the center of mass due to the retrofitting of the engine with additional mounted units, as well as the location of the mounting points of the supports on the vibrations of the power unit on the suspension at the design stage.

\section{REFERENCES}

[1] P. V. Safronov. Modeling of fluctuations of the engine on a suspension bracket on the modes with high cyclic instability, Ph.D. dissertation, MADI, Moscow, 1999.

[2] V. L. Biderman. Applied theory of mechanical vibrations, Moscow, Lenand, 2017, $416 \mathrm{p}$.

[3] G. Korn, T. Korn. Handbook of mathematics for scientists and engineers, Saint-Petersburg, Lan', 2003, 832 p.

[4] V. E. Tol'skij, L. V. Korchemnyj, G. V. Latyshev, L. M. Minkin. Fluctuations of the power unit of the car, Moscow, Mashinostroenie, 1976, $266 \mathrm{p}$.

[5]V.E. Tol'skij. Vibroacoustics of the car, Moscow, Mashinostroenie, 1988, $270 \mathrm{p}$.

[6] P. V. Safronov, M. A. Karpov. Method for calculating the dynamic behavior of a four-cylinder engine on elastic supports when idling, Vestnik Moskovskogo avtomobil'no-dorozhnogo gosudarstvennogo tehnicheskogo universiteta (MADI), no. 3 (14), pp. 26-32, 2008.

[7] M. G. Shatrov, I. V. Alekseev, K. A. Morozov, A. S. Hachijan, S. A. Prishvin, L. N. Golubkov et al. Automobile engines, Moscow, Akademija, 2010, $464 \mathrm{p}$.

[8] V. N. Lukanin, M. G. Shatrov, I. V. Alekseev, K. A. Morozov, A. S. Hachijan, S. A. Prishvin et al. Internal combustion engines. Theory of working processes, Moscow, Vysshaya shkola, 2010, $479 \mathrm{p}$. 